\title{
HIGHLIGHTS
}

LUPUS NEPHRITIS

\section{Urinary NGAL predicts renal flares in lupus nephritis}

Urinary neutrophil gelatinase-associated lipocalin (NGAL) level may predict disease activity in adult patients with lupus nephritis, according to new findings. "We found that levels of NGAL in the urine ... were a significant predictor of flare in patients with a history of biopsy-proven nephritis", explains the study's lead researcher Chaim Putterman.

Lupus nephritis is a common manifestation of systemic lupus erythematosus (SLE) and is associated with considerable morbidity and mortality. Early diagnosis of disease activity in the kidney may lead to improved outcomes in affected patients; however, available markers that can be measured noninvasively have limited specificity and sensitivity for predicting the development of renal disease in patients with SLE.

Previous studies have demonstrated that NGAL level increases in response to a variety of renal insults. Prasad
Devarajan and colleagues also previously demonstrated that levels of urinary NGAL correlate with renal damage in patients with childhood-onset SLE. Similarly, Putterman and colleagues have demonstrated that patients with lupus nephritis have higher urinary levels of NGAL than SLE patients without nephritis. These findings led Putterman's group to investigate in the current study whether high urinary NGAL levels could predict future renal disease activity.

In an observational study of $103 \mathrm{New}$

Yorkers with SLE, the researchers found that level of urinary NGAL was a significant predictor of future renal disease activity, defined as a Systemic Lupus Erythematosus Disease Activity Index (SLEDAI) score $\geq 4$. Moreover, in patients with biopsy-proven lupus nephritis, urinary NGAL was a significant predictor of renal flare. "Importantly, the performance of urinary NGAL as a predictor of renal flare in patients with biopsy-proven nephritis exceeded that provided by serum levels of anti-double-stranded DNA antibodies, a serologic marker in common clinical use for this purpose", says Putterman. The researchers also validated their findings against an independent cohort of SLE patients from London, in whom renal disease activity was determined by use of the British Isles Lupus Assessment Group (BILAG) index. "These findings are important because the London cohort had a very different ethnic breakdown from the New York cohort and a very different definition for scoring renal disease", states Putterman.

The researchers are currently conducting a large prospective study to confirm the role of urinary NGAL as a biomarker of lupus nephritis in adults.

Susan J. Allison

Original article Rubinstein, T. et al. Urinary neutrophil gelatinase-associated lipocalin as a novel biomarker for disease activity in lupus nephritis. Rheumatology (Oxford) doi:10.1093/rheumatology/kep468 